\title{
Designing LED array for uniform illumination based on local search algorithm
}

P. Lei

leipldu@163.com

Q. Wang

H. Zou
School of Information and Electric Engineering, Ludong University, Yantai, P.R.China

School of Information and Electric Engineering, Ludong University,,Yantai, P.R.China

We propose a numerical optimization method based on local search algorithm to design an LED array for a highly uniform illumination distribution. In the first place, an initial LED array is randomly generated and the corresponding value of the objective function is calculated. In the second place, the value of the objective function is iteratively improved by applying local changes of the LED array until the objective function value can not be improved. This method can automatically design an array of LEDs with different luminous intensity value and distribution. Computer simulations show that the near-optimal LED array with highly uniform illumination distribution on target plane is obtained by this method.

[DOI: http://dx.doi.org/10.2971/jeos.2014.14014]

Keywords: LED, design, uniform illumination, algorithm

\section{INTRODUCTION}

LEDs are gradually replacing traditional light sources in applications, such as road lighting, automotive lighting, indoor lighting and backlighting, due to LEDs' advantages of low energy consumption, long lifetime, small size and various colors [1]-[3]. However, in most cases, LEDs cannot meet the requirements because of the Lambertian radiation distribution. Secondary optical components such as freeform lens are designed for a single LED [4, 5]. Since one LED cannot provide sufficient power, several LEDs must be mounted on panels to enhance irradiance. A key problem is to achieve a highly uniform illumination distribution emitted by an LED array. I. Moreno proposed a method for optimizing LED-to-LED spacing to achieve uniform irradiance by considering each LED as an imperfect Lambertian source [6]. Z. Qin designed freeform lens with large view angle for LED uniform illumination [7]. W. Whang presented a method designing LED array with surface-tailored lens to obtain the desired LED emission angle for uniform illumination distribution [8]. J. Tian proposed trial and error method for obtaining the optimum LED-toLED spacing [9]. K. Wang optimized light intensity distribution curve and designed corresponding optical components to achieve uniform illumination when the distance-height ratio is given [10]. H. Yang presented an approach to demonstrate different uniform illumination patterns of LED array across a range of distances [11]. The methods above are all analytical methods and are based on Sparrow's criterion. Only the central point of target plane is constrained by Sparrow's criterion, so the methods cannot guarantee uniform illumination distribution across the whole target plane $[7,10]$. Because of great complexity of the analytical method, it is necessary to assume regular LED arrays and identical luminous inten- sity profiles of LEDs to achieve uniform illumination distributions. Z. Su proposed a numerical method for optimizing arrays of LEDs with different luminous intensity values and distributions [12]. However, the method takes too much time to find the optimal solution since all the candidate solutions in his method are randomly generated. With the increase of iterative times, the probability of getting a better candidate solution decreases seriously, so the newly generated solutions have to be discarded because of their worse values. In this paper, we design a numerical optimization algorithm to arrange the LED array for uniform illumination distribution on target plane. We employ local search algorithm to optimize the position of each LED in the array by minimizing the objective function. We firstly construct an objective function which effectively represents the uniformity of the illumination distribution, and then minimize the objective function so as to obtain the highly uniform illumination on target plane.

Local search is a heuristic method for solving numerous hard optimization problems $[13,14]$. Local search algorithm starts from a random initial solution which is iteratively improved by moving to a neighbor in the space of candidate solutions. The algorithm terminates when no better solutions can be found or a time bound is elapsed.

The article is arranged as follows. The principle of highuniform LED array design is introduced in Section 2. The algorithm based on local search is presented in Section 3. The computer simulation results are given in Section 4. Finally we draw conclusions in Section 5. 


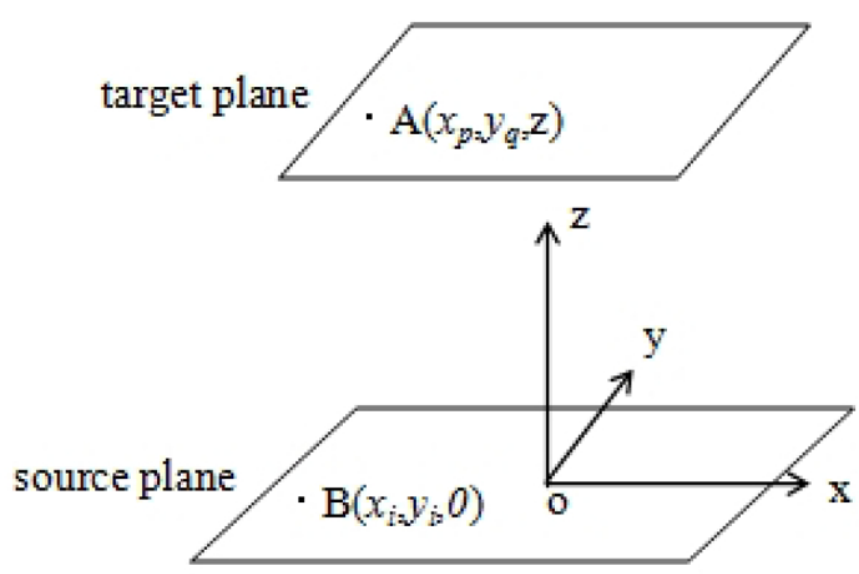

FIG. 1 Points on S-plane and T-plane with Cartesian coordinates.

\section{PRINCIPLE OF HIGH-UNIFORM LED ARRAY DESIGN}

An ideal LED is a Lambertian emitter, i.e., its luminous intensity distribution is a cosine function of the view angle. In practice, the luminous intensity of a single LED can be treated approximately as imperfect Lambertian distribution given by $[6,15]$

$$
I(\theta)=I_{0} \cos ^{m} \theta,
$$

where $I_{0}$ is luminous intensity at the normal direction to the source surface and $\theta$ is the view angle. The number $m$ is given by the angular half width $\theta_{1 / 2}$ (a value provided by the manufacturer, defined as the view angle when irradiance is the half of the value at the normal direction):

$$
m=-\ln 2 / \ln \left(\cos \theta_{1 / 2}\right) \text {. }
$$

To design a practical tool, we only analyze the irradiance distribution over a target plane (T-plane) in parallel with the surface of the LED array. The irradiance distribution across target plane from a single LED at source plane(S-plane) can be described with Cartesian coordinates. The irradiance at the point $A$ on T-plane with coordinates $\left(x_{p}, y_{q}, z\right)$ from the $i$-th LED in the array at the point $B$ on S-plane with coordinates $\left(x_{i}, y_{i}, 0\right)$ (see Figure 1) is expressed as

$$
\begin{aligned}
E_{i}\left(x_{p}, y_{q}, z\right) & =\frac{I\left(\theta_{i}\right) \cos \theta_{i}}{r_{i}^{2}} \\
& =\frac{I\left(\theta_{i}\right) z}{\left[\left(x_{i}-x_{p}\right)^{2}+\left(y_{i}-y_{q}\right)^{2}+z^{2}\right]^{3 / 2}},
\end{aligned}
$$

where $z$ is the distance between target plane and source plane. The irradiance at the point $\left(x_{p}, y_{q}, z\right)$ with an array of $n$ LEDs is given by

$$
E\left(x_{p}, y_{q}, z\right)=\sum_{i=1}^{n} E_{i}\left(x_{p}, y_{q}, z\right) .
$$

We divide the target plane and source plane into $L_{t} \times W_{t}$ and $L_{S} \times W_{S}$ grids respectively as shown in Figure 2 .

We assume that each LED is placed at the central point of the grid on source plane. The irradiance at the central point of each grid on target plane is calculated by Eq. (4) to obtain the

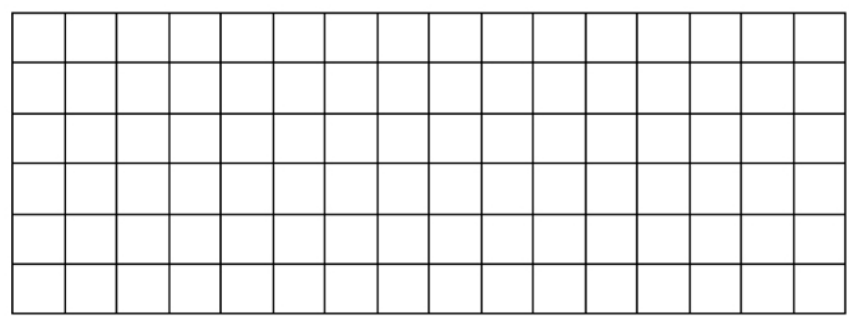

FIG. 2 Target and source planes with grids.

objective function value. There are several methods to represent the illumination uniformity of an LED array $[9,16]$. We use the $C V$ (RMSE), which is the abbreviation of coefficient of variation of root mean square error, as the objective function to evaluate the illumination uniformity on the target plane $[10,12]$. The objective function is denoted by

$$
f\left(x_{1}, y_{1}, \ldots, x_{i}, x_{i}, \ldots, x_{n}, x_{n}\right)=\sigma / \bar{E}
$$

where $\sigma$ is the standard error of irradiance of all grids, which is expressed by

$$
\sigma=\sqrt{\sum_{p=1}^{W_{t}} \sum_{q=1}^{L_{t}}\left(E\left(x_{p}, y_{q}, z\right)-\bar{E}\right)^{2} /\left(L_{t} \times W_{t}\right)},
$$

and $\bar{E}$ denotes the average irradiance of all grids, which is given by

$$
\bar{E}=\frac{1}{L_{t} \times W_{t}} \sum_{p=1}^{W_{t}} \sum_{q=1}^{L_{t}} E\left(x_{p}, y_{q}, z\right)
$$

The irradiance $E\left(x_{p}, y_{p}, z\right)$ of Eq. (6) is calculated by Eq. (4). Different LED arrays usually generate different objective function values. We minimize the objective function in order to get the optimal LED array arrangement for highly uniform illumination distribution on the target plane.

\section{ALGORITHM DESIGN}

We will explain how to optimize an LED array by local search algorithm. The steps of the algorithm (named LSUniform) is described as follows.

LSUniform algorithm:

(1) Initialization

(1.1) A vector $s$ is set to store the solution. The vector con- sists of $2 n$ elements representing the coordinates of $n$ LEDs. Let $\boldsymbol{s}^{*}$ represent the best solution.

(1.2) The objective function is constructed to reflect the illumination uniformity of LED array.

(1.3) A Boolean variable flag denotes whether the objective function value is improved or not. Set flag = false.

(1.4) Generate a random initial solution as the current best solution $\boldsymbol{s}^{*}$ and calculate the objective function value $f\left(s^{*}\right)$. 
(2) Repeat

(2.1) Set $k=1$.

(2.2) Search in the neighbor of the $k$-th LED for the better position on the S-plane.

(2.2.1) Examine the right position of the $k$-th LED: Along the positive direction of $x$ axis, find the first empty position of $k$-th LED or no empty position is found due to reaching the edge of the S-plane. If the $k$-th LED is moved to the empty position, then a new solution $\boldsymbol{s}_{1}$ will be generated.

(2.2.2) Examine the left position of the $k$-th LED: Along the negative direction of $x$ axis, do the same as step (2.2.1) and generate a solution $\boldsymbol{s}_{2}$.

(2.2.3) Examine the up position of the $k$-th LED: Along the positive direction of $y$ axis, do the same as step (2.2.1) and generate a solution $\boldsymbol{s}_{3}$.

(2.2.4) Examine the down position of the $k$-th LED: Along the negative direction of y axis, do the same as step (2.2.1) and generate a solution $\boldsymbol{s}_{4}$.

(2.2.5) Calculate the objective function values $f\left(s_{i}\right)$ $(i=1,2,3,4)$ to get the minimum of $f\left(s_{i}\right)$. The solution $s^{\prime}$ corresponding to the minimum value of $f\left(s_{i}\right)$ is obtained.

(2.2.6) if $f\left(s^{\prime}\right)<f\left(s^{*}\right)$, then $s^{*}=s^{\prime}$, move the $k$-th LED to the empty position corresponding to $s^{\prime}$ and set flag $=$ true.

(2.3) $k=k+1$. if $k<n$, then go back to (2.2).

(2.4) if flag $==$ true, then go back to (2.1).

(3) Output the best solution $\boldsymbol{s}^{*}$.

LSUniform algorithm first generates a random initial solution as the current best $\boldsymbol{s}^{*}$ and then apply the following procedures to decide which position the $k$-th $\operatorname{LED}(k=1,2, \ldots, n)$ will move to. The $k$-th LED can move left, right, up and down, along the directions of $x$ and $y$ axis. Along each direction, after examining the first empty position where no LED is installed, LSUniform can get a corresponding solutions $\boldsymbol{s}_{i}(i=1,2,3,4)$ as well as the objective function values $f\left(s_{i}\right)$. Then, the best solution $\boldsymbol{s}^{\prime}$ corresponding to the minimum value of $f\left(\boldsymbol{s}_{i}\right)$ can be obtained. If $f\left(s^{\prime}\right)<f\left(s^{*}\right)$, we move the $k$-th LED to the position corresponding to the solution $\boldsymbol{s}^{\prime}$ and set flag $=$ true. Steps are described in the flowing chart of LSUniform algorithm in Figure 3. In order to avoid getting stuck in local minima, we should run LSUniform algorithm several times with different initial solutions and select the best solution. We use $T$ to denote the count of calling LSUniform algorithm. We use $\alpha$ to denote the maximum count of running times of the steps (2.1) through (2.4) if LSUniform runs $T$ times. Now we analyze the computational time complexity of LSUniform algorithm. Let $N_{t}=L_{t} \times W_{t}, N_{s}=L_{s} \times W_{s}$. Step (1.4) runs in $n \cdot N_{s}+n \cdot N_{t}$ times. Steps (2.1) through (2.4) run in $\alpha \cdot n \cdot N_{t}$ times. Hence, the time complexity of the LSUniform is $\mathrm{O}\left(n \cdot N_{s}+\alpha \cdot n \cdot N_{t}\right)$ in the worst case. In the following simulations, $\alpha$ is less than 50 , so the LSUniform algorithm is time-saving.

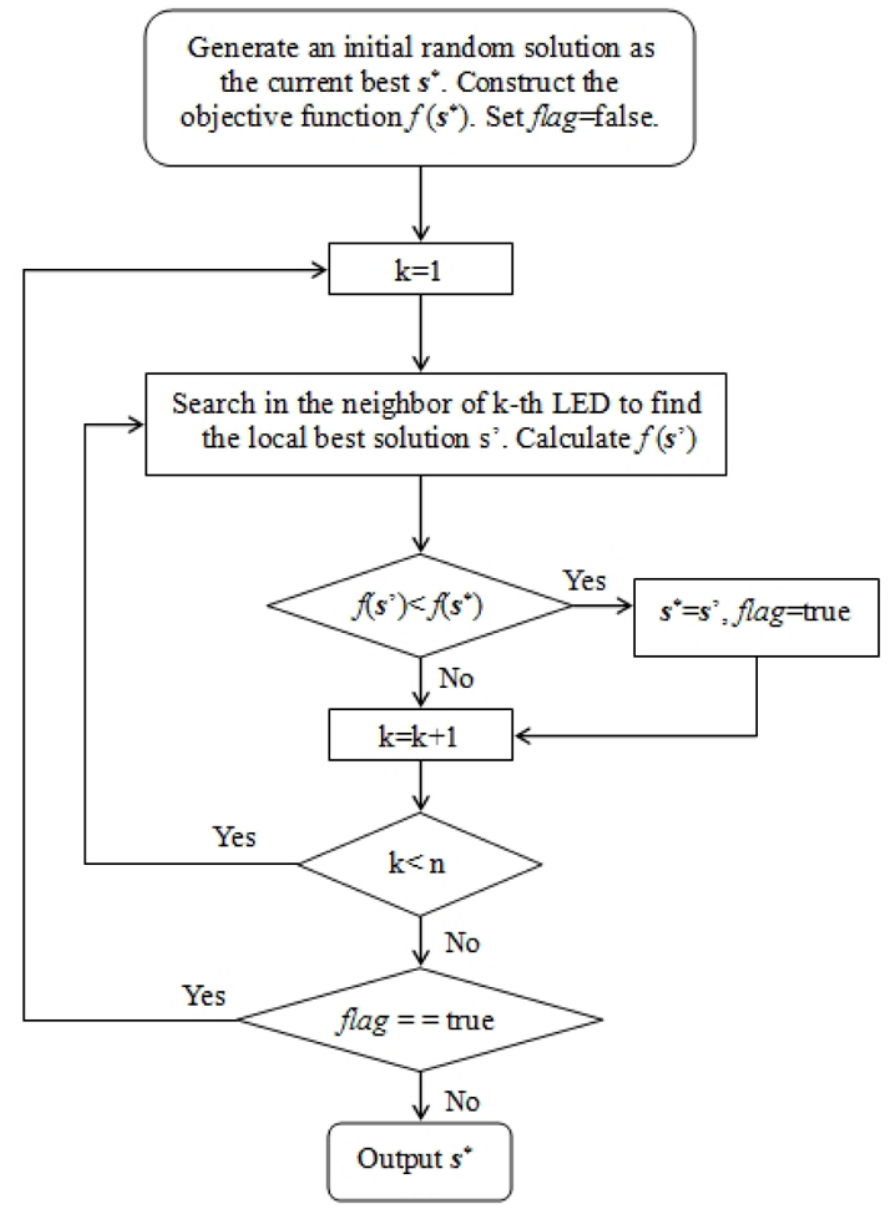

FIG. 3 Flowing chart of LSUniform algorithm.

\section{COMPUTER SIMULATIONS}

We design three arrays of LEDs with different luminous intensity distributions in order to verify the efficiency of our method.

\subsection{LED with perfect Lambertian luminous intensity distribution}

The first LED array includes 7 LEDs, each of which has perfect Lambertian luminous intensity distribution. The 7 LEDs are divided into two types. The first type consists of 3 LEDs, and the other type consists of 4 LEDs. The intensity value of each LED in the first type is 1.5 times as much as that of each LED in the other type. As shown in Figure 4, the solid curve represents the intensity value of the first type, and the dashed curve represents that of the other. The size of the source plane is the same as the size of the target plane in the simulation. We call LSUniform 20 times and the maximum count $\alpha$ of steps (2.1) through (2.4) equals to 45 . To compare the result with Su's [12], $C V_{S U}$ and $C V_{L S}$ denote the values of the objective function of Su's method and LSUniform respectively. The simulation result of our method and the result of Su's are shown in Table 1. It is shown in Table 1 that the result of our method is better than that of Su's. After optimization, the optimized arrangement and the relative irradiance map of the first array are shown in Figure 5 and Figure 6 respectively. In Figure 5, the blue circles and the red diamonds represent the LEDs cor- 


\begin{tabular}{|c|c|c|c|c|c|}
\hline Target plane size & Distance between source and target plane & Times of running LSUniform & $\alpha$ & $C V_{L S}$ & $C V_{S U}$ \\
\hline $40 \times 40(\mathrm{~cm})$ & $50(\mathrm{~cm})$ & 20 & 45 & 0.044 & 0.096 \\
\hline
\end{tabular}

TABLE 1 Result of our method and that of Su's method for uniform illumination distribution.

\begin{tabular}{|c|c|c|c|c|c|c|c|c|}
\hline LED number & $z(\mathrm{~cm})$ & $d(\mathrm{~cm})$ & $\begin{array}{c}\text { Times of running } \\
\text { LSUniform }\end{array}$ & $\begin{array}{c}\text { Source plane } \\
\text { size }\left(\mathrm{cm}^{2}\right)\end{array}$ & $\begin{array}{c}\text { Target plane } \\
\text { size }\left(\mathrm{cm}^{2}\right)\end{array}$ & $\alpha$ & $C V_{L S}$ & $C V_{\text {Moreno }}$ \\
\hline \multirow{6}{*}{36} & \multirow{6}{*}{24} & \multirow{6}{*}{5} & \multirow{6}{*}{50} & \multirow{6}{*}{$30 \times 30$} & $30 \times 30$ & 13 & 0.028 & 8.362 \\
\hline & & & & & $26 \times 26$ & 12 & 0.015 & 4.596 \\
\hline & & & & & $22 \times 22$ & 14 & 0.011 & 2.204 \\
\hline & & & & & $18 \times 18$ & 15 & 0.006 & 0.903 \\
\hline & & & & & $14 \times 14$ & 15 & 0.003 & 0.311 \\
\hline & & & & & $10 \times 10$ & 17 & 0.001 & 0.087 \\
\hline
\end{tabular}

TABLE 2 Result of our method and that of Su's method for uniform illumination distribution.

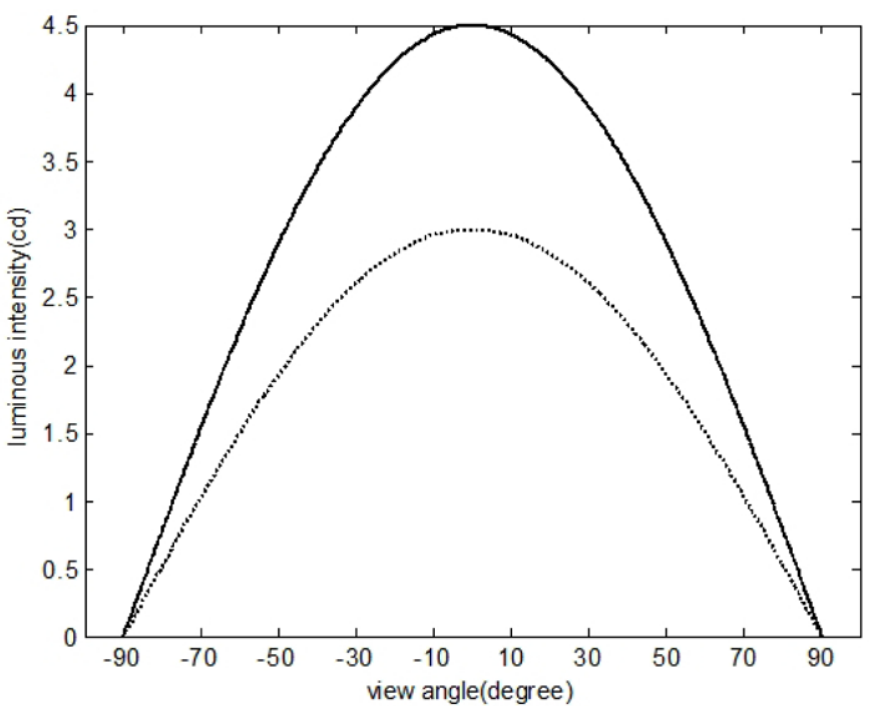

FIC. 4 The luminous intensity distribution of different types of LEDS.

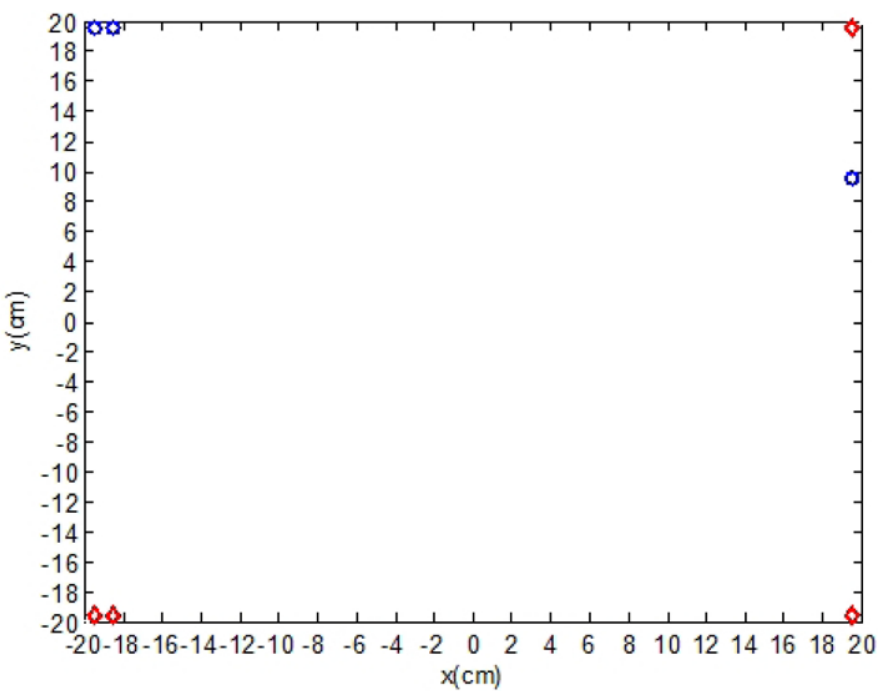

FIG. 5 The optimized arrangement of the first LED array.

responding to the solid intensity distribution curve and the dashed intensity distribution curve in Figure 4, respectively.

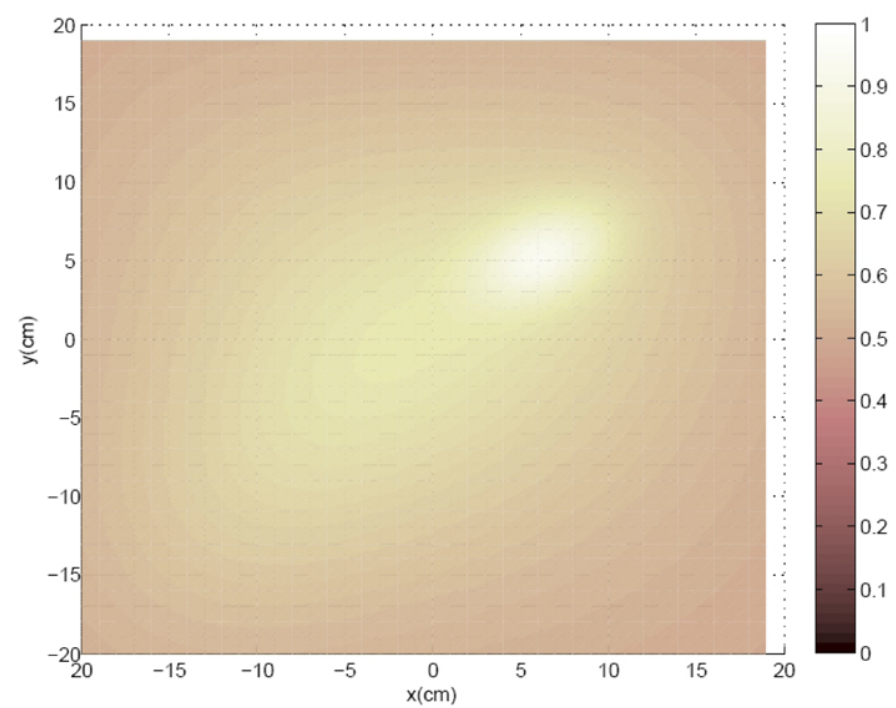

FIG. 6 The relative irradiance map of the first array.

\subsection{LED with imperfect Lambertian luminous intensity distribution}

The second array consists of 36 LEDs and each LED has identical imperfect Lambertian intensity distribution and value. For arrays larger than $4 \times 4$ LEDs $(m>30)$, Moreno gave an empirical formula $\mathrm{d} / \mathrm{z}=\sqrt{1.2125 /(m-3.349)}$, where $d$ is the optimum LED-to-LED spacing of LED array. Figure 7 shows the luminous intensity distribution with $\mathrm{m}=31.37$ in Eq. (1). To compare our method with Moreno's, let $C V_{\text {Moreno }}$ represent Moreno's results. When the parameter $m$ of each LED equals to 31.37, the results of our method and Moreno's are shown in Table 2 with the invariable size of the source plane and the various sizes of the target planes. It's clear that our results are much better than Moreno's. After optimization, the optimized arrangement and the irradiance map of the second array are shown in Figure 8 and Figure 9 respectively when the target plane size is $10 \times 10\left(\mathrm{~cm}^{2}\right)$.

\subsection{LED with arbitrary luminous intensity values and distributions}

The parameters of angle distribution of LEDs are usually provided by manufacturers. Freeform lens can be used to freely redistribute light flux from an LED [8]. The intensity distribu- 


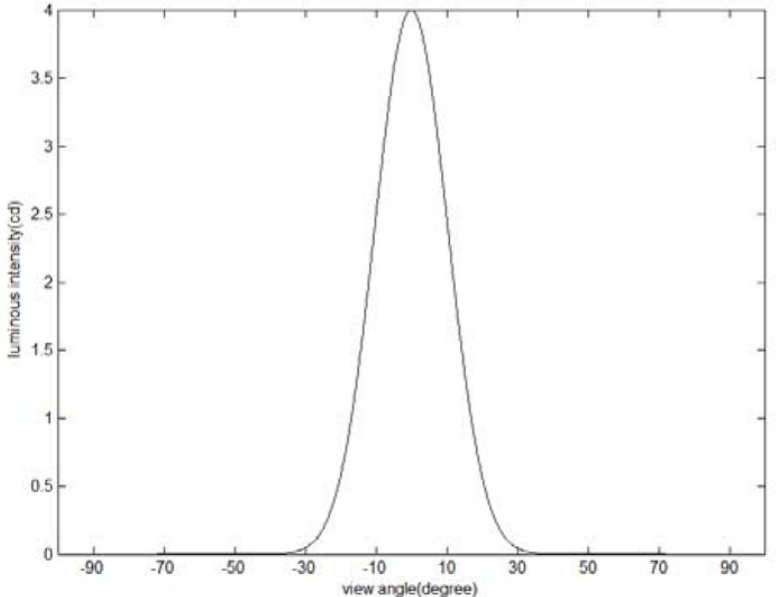

FIG. 7 The imperfect Lambertian luminous intensity distribution of the LED when $\mathrm{m}=31.37$.

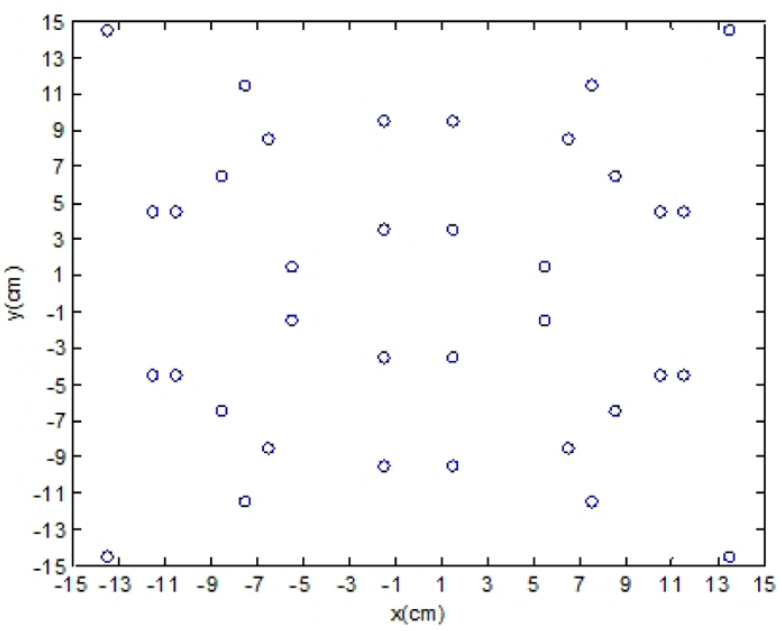

FIG. 8 The optimized arrangement of the second LED array when the target plane size is $10 \times 10\left(\mathrm{~cm}^{2}\right)$

tion of each LED in the third array is given by Wang [10]:

$$
I(\theta)=a_{0}+a_{1} \theta^{2}+a_{2} \theta^{4}+a_{3} \theta^{6}+a_{4} \theta^{8},
$$

where $a_{0}=1.0, a_{1}=0.878827, a_{2}=1.0, a_{3}=0.8, a_{4}=-0.8$. Figure 10 shows the luminous intensity distribution as given in Eq. (8). We use $C V_{\text {Wang }}$ to denote Wang's results. The compared results of our method and Wang's are shown in Table 3. It can be seen in Table 3 that our results are better than Wang's. Figure 11 shows the optimized LED arrangement and the corresponding irradiance map is shown in Figure 12 when the target plane size is $28 \times 28\left(\mathrm{~cm}^{2}\right)$.

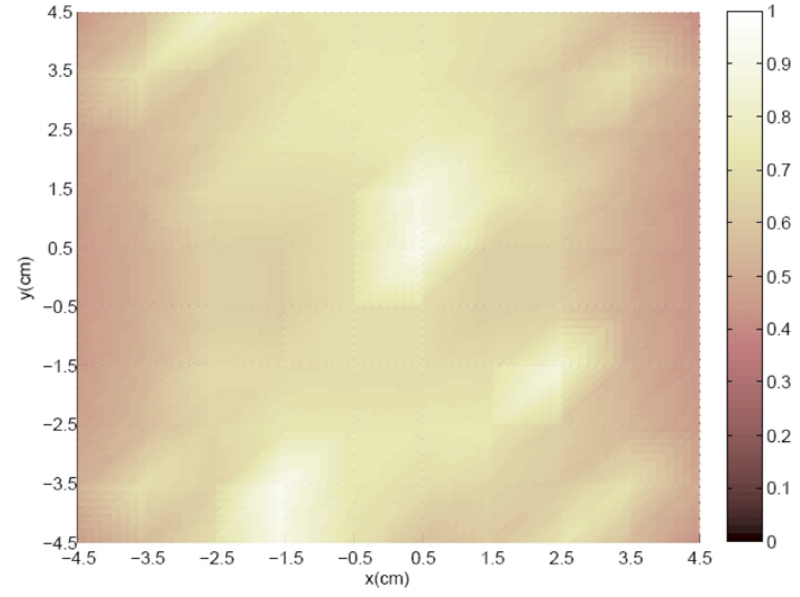

FIG. 9 The relative irradiance map of the second array when the target plane is $10 \times 10\left(\mathrm{~cm}^{2}\right)$

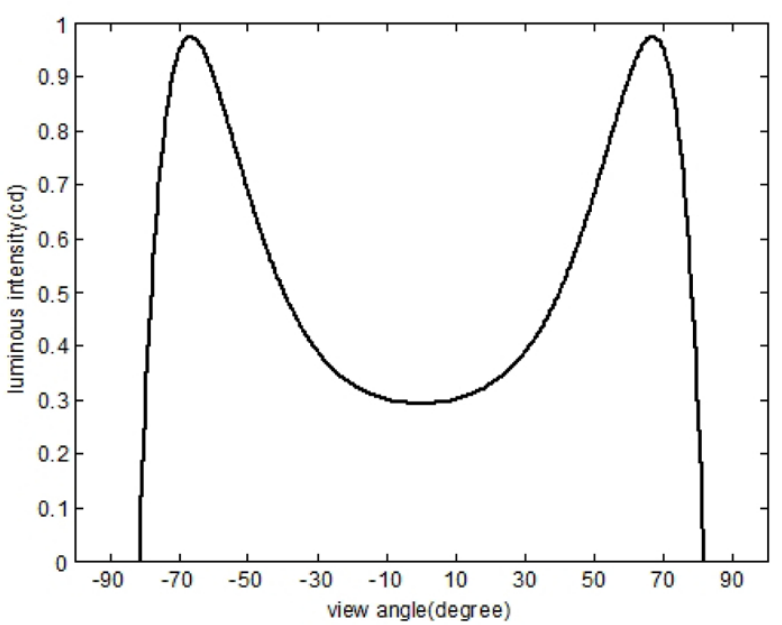

FIG. 10 The luminous intensity distribution of the third array of LEDs.

\section{CONCLUSION}

In this study, we design a method based on local search to optimize the LED array for highly uniform illumination distribution. We use three different LEDs to demonstrate the efficiency of our method. We compare our method both with the analytical and numerical methods under the condition that each LED in the array has the same or different luminous intensity values and distributions. The simulation results prove that the method we proposed is more accurate and time- saving.

\begin{tabular}{|c|c|c|c|c|c|c|c|c|}
\hline LED number & $z(\mathrm{~cm})$ & $d(\mathrm{~cm})$ & $\begin{array}{l}\text { Times of running } \\
\text { LSUniform }\end{array}$ & $\begin{array}{c}\text { Source plane } \\
\operatorname{size}\left(\mathrm{cm}^{2}\right)\end{array}$ & $\begin{array}{c}\text { Target plane } \\
\text { size }\left(\mathrm{cm}^{2}\right)\end{array}$ & $\alpha$ & $C V_{L S}$ & $C V_{\text {Wang }}$ \\
\hline \multirow{6}{*}{36} & \multirow{6}{*}{3} & \multirow{6}{*}{6} & \multirow{6}{*}{40} & \multirow{6}{*}{$30 \times 30$} & $30 \times 30$ & 16 & 0.034 & 0.095 \\
\hline & & & & & $28 \times 28$ & 14 & 0.025 & 0.070 \\
\hline & & & & & $26 \times 26$ & 16 & 0.019 & 0.051 \\
\hline & & & & & $24 \times 24$ & 14 & 0.016 & 0.050 \\
\hline & & & & & $22 \times 22$ & 19 & 0.015 & 0.037 \\
\hline & & & & & $20 \times 20$ & 14 & 0.011 & 0.019 \\
\hline
\end{tabular}




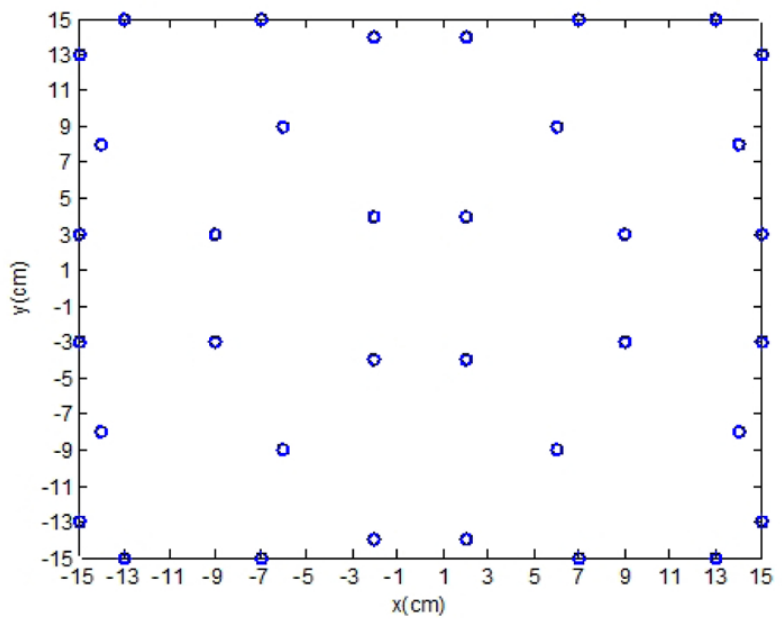

FIC. 11 The optimized arrangement of the third LED array when the target plane size is $28 \times 28\left(\mathrm{~cm}^{2}\right)$.

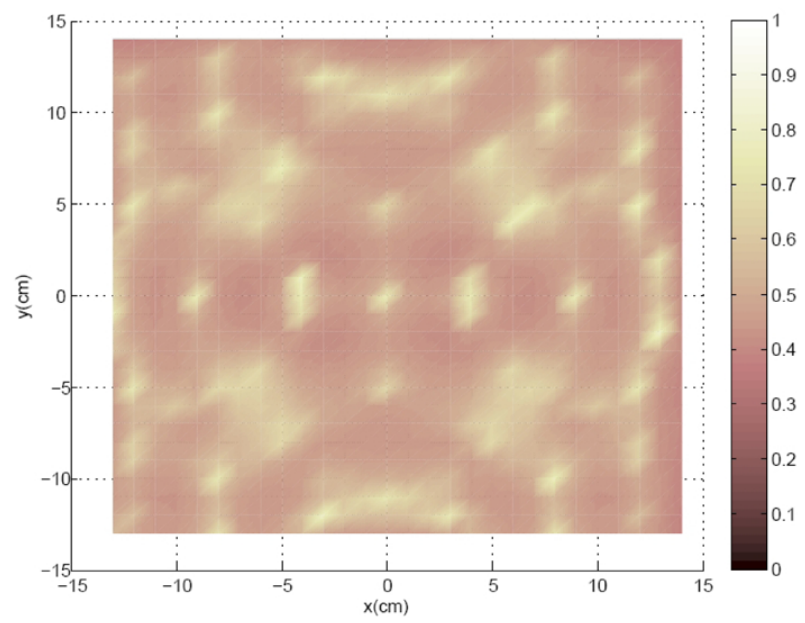

FIG. 12 The relative irradiance map of the third array when the target plane is $28 \times 28\left(\mathrm{~cm}^{2}\right)$.

\section{ACKNOWLEDGEMENTS}

This work was supported by the National Natural Science Foundation of China (No.61170161).

\section{References}

[1] E. F. Schubert, J. K. Kim, "Solid-state light sources getting smart," Science 308, 1274-1278 (2005)

[2] Y. Narukawa, "White-light LEDs," Opt. Photonics News 15, 24-29 (2004).
[3] J. Kovác, J. Jakabovic, and M. Kytka, "Advanced light emitting devices for optoelectronic applications," Proc. SPIE 7138, 71382A (2008).

[4] K. Wang, S. Liu, F. Chen, Z. Qin, Z. Y. Liu, and X. B. Luo, "Freeform LED lens for rectangularly prescribed illumination," J. Opt. A-Pure Appl. Opt. 11, 105501 (2009).

[5] Z. X. Feng, Y. Luo, and Y. J. Han, "Design of LED freeform optical system for road lighting with high luminance/illuminance ratio," Opt. Express 18, 22020-22031 (2010).

[6] I. Moreno, M. Avendaño-Alejo, and R. I. Tzonchev, "Designing lightemitting diode arrays for uniform near field irradiance," Appl. Optics 45, 2265-2272 (2006).

[7] Z. Qin, K. Wang, F. Chen, X. B. Luo, and S. Liu, "Analysis of condition for uniform lighting generated by array of light emitting diodes with large view angle," Opt. Express 18, 17460-17476 (2010).

[8] A. J. W. Whang, Y. Y. Chen, and Y. T. Teng, “Designing uniform illumination systems by surface-tailored lens and configurations of LED arrays," J. Disp. Technol. 5(3), 94-103 (2009).

[9] J. Tan, K. Yang, M. Xia, and Y. Yang, "Analysis of uniform illumination system with imperfect Lambertian LEDs," Opt. Appl. 41, 507-517 (2011).

[10] K. Wang, D. Wu, Z. Qin, F. Chen, X. B. Luo, and S. Liu, "New reversing design method for LED uniform illumination," Opt. Express 19, 830-840 (2011).

[11] H. Yang, J. W. M. Bergmans, T. C. W. Schenk, J. P. M. G. Linnartz, and R. Rietman, "Uniform illumination rendering using an array of LEDs: a signal processing perspective," IEEE T. Signal Proces. 57, 1044-1057 (2009).

[12] Z. Su, D. Xue, and Z. Ji, “Designing LED array for uniform illumination distribution by simulated annealing algorithm," Opt. Express 20, 843-855 (2012).

[13] H. R. Lourenço, 0. Martin, and T. Stützle, "Iterated Local Search: Framework and Applications," Int. Ser. Oper. Res. Man. 146, 363-397 (2010).

[14] E. Arkin, and R. Hassin, "On local search for weighted k-set packing," Math. Oper. Res. 23, 640-648 (1998).

[15] D. Wood, Optoelectronic Semiconductor Devices (Prentice-Hall International, New Jersey, 1994).

[16] I. Moreno, "Illumination uniformity assessment based on human vision," Opt. Lett. 35, 4030-4032 (2010). 